\title{
The Impact of Peripheral Venous Catheter Procedural Kits on Improving Clinical Outcomes in Hospitalised Patients
}

\author{
Liam Callaghan ${ }^{1}$, Paul McCarron ${ }^{1}$, Michael Scott ${ }^{2}$, Pat McKee ${ }^{2}$, Geraldine Conlon-Bingham ${ }^{2}$, David Farren ${ }^{2}$, \\ Alan Tate ${ }^{3}$, Stuart Murray ${ }^{3}$, Eoin Dunne ${ }^{3}$ and Kathryn Burnett ${ }^{1 *}$ \\ ${ }^{1}$ Department of Pharmacy and Pharmaceutical Sciences, Ulster University, United Kingdom \\ ${ }^{2}$ Northern Health and Social Care Trust, Ballymena, United Kingdom \\ ${ }^{3}$ Iskus Health Ltd, Ireland
}

*Corresponding author: Kathryn Burnett, School of Pharmacy and Pharmaceutical Sciences, Ulster University, Cromore Road, Coleraine, Northern Ireland, United Kingdom.

Received Date: July 13, 2019

Published Date: July 17, 2019

\section{Abstract}

Introduction: Peripheral Venous Catheters (PVC) are an essential component in modern healthcare and their inclusion into a Clinical Procedure Pack has been identified as an area of healthcare where an enhancement in design and implementation could improve practitioner performance and associated patient outcomes.

Aim: The aim of this study was to assess the clinical impact of two interventions;

i. procedural kits with an enhanced training programme

ii. a specific feedback mechanism on the rates of inappropriate removal of PVCs within Antrim Area Hospital.

Methods: A time series design, using retrospective and prospective data was used. The study consisted of three phases. Phase A aimed to establish existing PVC adverse event rates. Phase B included the introduction and assessment of the first intervention, a PVC procedural kit and associated training package, to specific clinical areas. Phase $\mathrm{C}$ involved the introduction and assessment of the second intervention, a performance feedback mechanism.

Results: Analysis of data showed that the difference of mean PVC clinical adverse event rate between Phase A 12.84\% (95\% CI: 10.86 - 15.03) and Phase B of 9.48\% (95\% CI: 8.10 - 11.00) was improved ( $\mathrm{p}=0.008)$. Similarly, the PVC clinical adverse event rate of Phase B of $9.48 \%(95 \%$ CI: 8.10 - 11.00) and Phase C of 5.94\% (95\% CI: $4.78-7.30)$, was improved $(\mathrm{P}<0.001)$.

Conclusion: In conclusion, the study demonstrated the benefit of introducing a PVC procedural kit with an enhanced training programme and a specific feedback mechanism to significantly reduce clinical adverse events, compared to the previous standard practice.

Keywords: PVC; Adverse events; Procedural pack; Clinical outcomes

Abbreviations: AAH: Antrim Area Hospital; AE: Adverse Event; ANOVA: One-Way Analysis of Variance; ANTT: Aseptic Non-Touch Technique; BSI: Bloods Stream Infection; FY1: Foundation Year one Doctor; ICU: Intensive Care Unit; IQR: Interquartile range; IV: Intravenous; NHS: National Health Service; NHSCT: Northern Health and Social Care Trust; OSCE: Objective Structured Clinical Examination; PCIM: Peripheral Cannula Insertion and Management Form; PVC: Peripheral Venous Catheter; SPC: Statistical Process Control

\section{Introduction}

Peripheral Venous Catheters (PVC) are an essential component in modern healthcare, with approximately a third of all UK and US inpatients having at least one device inserted during their hospital stay [1,3]. Peripheral venous cannulation carries a number of risks to both patient and health care staff, resulting in additional workload and increased costs [4,5]. A 2015 study reported that $59 \%$ of patients worldwide had at least one PVC in situ during an international prevalence study involving 14 countries [6]. It is estimated that up to $80 \%$ of patients require at least one PVC to be inserted during their hospital stay [7]. 
The routine use of PVCs may have led to a certain amount of triviality associated with complications resulting from the procedure [3]. Unplanned PVC restarts are a result of both clinical and non-clinical events, with an overall failure rate ranging from $33 \%$ to $69 \%[4,5,8]$. Failure of a single PVC in a patient can result in a negative cycle of catheter removal and reinsertion, increasing risk of complications and cost [9]. Problems associated with the procedure can be broken into major and minor complications.

Major complications result in a new diagnosis as a consequence of the insertion, maintenance or removal procedure. These major complications include blood stream infection, phlebitis, infiltration and extravasation $[10,11]$. The reported rates of major complications due to peripheral cannulation includes Blood Stream Infection (BSI) at $0.1 \%$, extravasation at $6 \%$, infiltration at $36.3 \%$ and phlebitis ranging from $2.1 \%$ up to $60 \%$ in some cases [1115]. The impact to the patient ranges from life-threatening and potential disability through to disruption of treatment and clinical deterioration [11]. Additional treatment, patient anxiety and increased costs are also associated with the occurrence of major complications [11]. While studies show that there are relatively low rates of infection associated with PVC access, it is thought to be an under-reported issue as IV therapy is not usually suspected as the source of the infection [12]. The perceived low rate of infection is still a major cause of concern when the large numbers of PVC devices used on a daily basis are taken into consideration [7].

Minor PVC complications can result in treatment disruption and patient anxiety, but do not cause a serious impact on patients' clinical wellbeing. Minor complications include dislodgements (accidental removal) and leaking [10,16]. A similar large variable range of rates exist with dislodgement rate ranging from $2.4 \%$ to $36 \%$ and leaking rates of $3.9 \%$ to $12.4 \%$ reported $[8,10,17]$. Complications resulting from PVC use can be assigned to factors relating to the patient or hospital environment [18]. There have been a number of studies aimed at reducing PVC associated adverse event rates, concentrating mainly on phlebitis and BSI $[1,19,20]$. A singular approach to PVC practice intervention has led to marginal quality improvement, with limited lasting effect $[19,21]$.

Clinical guidelines and well-designed care bundles have been shown to improve patient outcomes, however the implementation of these interventions can also cause problems [1,3,22]. Education and training are one of the main recommendations directed by guidelines for the prevention of intravascular catheter-related infections [18]. Focused educational interventions have been successful in reducing PVC related adverse events [23]. However, education alone seems to have a limited benefit on the reduction of overall adverse events associated with PVC insertion and longterm care [22,23]. The lack of appropriate equipment prior to a procedure has been shown to reduce compliance with care bundle practices [2]. Bespoke procedure packs promote adherence to the recommended procedural method, by ensuring the availability of all essential apparatuses [24,25]. When combined with training and familiarity of a pack, it allows the practitioner to concentrate on the technical aspects of the procedure while utilizing all equipment in the step by step manner in which the pack unfolds $[24,25]$. Procedural kits were identified as an area where an enhancement in design and implementation could improve practitioner performance and associated outcomes [2].

\section{Materials and Methods}

This study uses a multimodal intervention approach involving the introduction of a dedicated procedural kit, smallscale face to face teaching sessions, manikin training tools and direct feedback of error rates. A quasi-experimental time series design, using prospective data was used to evaluate the impact of two interventions on measured adverse events associated with initiation of peripheral venous catheters. The primary endpoint for the study was PVC failure, which was defined as a clinical adverse event resulting in the premature removal of PVC, prior to cessation of treatment. Suitable adverse event endpoints were identified and included blood stream infection, phlebitis, infiltration, and extravasation. Secondary endpoints included the measure of PVC non-clinical adverse events, classified as dislodgements and leaking. The measure of PVC adverse events recorded was compared to the total number of PVC recorded to completion (adverse events / number of PVCs assessed $\times 100$ ). The impact of the interventions was evaluated by comparing the pre- and post- mean PVC adverse event rate, as calculated through Statistical Process Control p-charts.

The study involved a 72-week period of data collection broken up into three phases, from July 2013 to April 2015. Phase A covered an initial 24-week period (July 2013 to December 2013), which was designed to establish existing PVC adverse event rates prior to the introduction of two interventions. Phase B covered an additional 24-week period (March 2014 to September 2014) and included the introduction and assessment of the first intervention, a PVC procedural kit and associated training package, to specific clinical areas. Phase C covered a further 24-week period (November 2014 to April 2015) designed to assess the second intervention, a feedback mechanism.

All adult inpatients in designated clinical medical and surgical wards, who had a PVC inserted and the device removed during their current hospital stay were included in the study. The inclusion criteria included PVCs inserted with correct associated documentation and data recorded to completion. Interventions were aimed at personnel who frequently performed the majority of PVC procedures, namely nursing staff and Foundation Year one (FY1) doctors. The project was classified as a service evaluation in accordance with the National Research Ethics Service 'Defining Research' guidelines and therefore did not require ethical review.

\section{Study setting}

This project was based within the Northern Health and Social Care Trust (NHSCT) in Northern Ireland, with Antrim Area Hospital (AAH) as the main site for ward-based data collection. Antrim Area Hospital is a 426 bedded district general teaching hospital, the largest in the NHSCT, providing services for a population of approximately 420,000. 


\section{Peripheral cannula insertion and management form}

A new Peripheral Cannula Insertion and Management (PCIM) Form was designed and introduced to the study clinical ward areas, before baseline data was collected. The form was developed by the NHSCT Infection Control Nursing team to improve records on the insertion, maintenance and removal of PVCs. It was additionally modified to record all pertinent information required for this study. The PCIM form provided an individual record for each PVC in-situ and improved accountability for each stage of insertion, on-going care and removal. It supported adherence to PVC guidelines due to the care bundle prompt checklist, colour coding and visual aids. The PCIM Form uses a colour coded observation frequency chart to allow the tracking of a PVC in-situ for up to 72 hours as standard practice, with an additional section available to extend the recording for a further 48 hours. The PVC on- going care bundle chart allowed recording for continuous and non-continuous infusions, which would lead to 6 or 12 hourly record entries. The form was beneficial to adverse events detection as a result of the integrated phlebitis scale, infiltration scale and extravasation guidance.

\section{Phases of study}

Phase A - baseline data: The first phase was carried out to determine the baseline pre-intervention AAH PVC adverse event rate using the standard insertion procedure, before any interventions were introduced. This included the use of up-todate evidence based guidelines, published from the Department of Health and the manual collection of components to complete the PVC procedure [26].

Phase B - intervention (PVC procedural kit and training programme): The PVC procedural kit was introduced to the clinical areas designated for the study. Training was provided on the use of the PVC procedural kit for all nurses and FY1 doctors who performed the PVC insertion procedure and were based on, or rotated through, the designated clinical wards. Phase B intervention training focused on correct use and application of the PVC procedural kit in conjunction with the importance of correct insertion procedure utilising an Aseptic Non-Touch Technique (ANTT) and completion of associated documentation. The PVC procedure training was delivered by members of the NHSCT Infection Control Nursing Team, with educational support material supplied by Iskus Health Ltd, followed by a practical component with Objective Structured Clinical Examination (OSCE) assessment.

Phase C - intervention (feedback mechanism): A results feedback mechanism was introduced to augment the PVC procedural kit and training programme in place from the previous intervention. Using continual accumulated data, practitioners displaying evidence of non- compliance (PVC adverse events) were identified. Direct feedback of results took the form of an email sent to the designated wards and to the FY1s who had completed the NHSCT PVC insertion-training programme. A general email was sent monthly to nurse ward managers from the specific wards. This email contained a run chart of AAH PVC adverse event rates broken into separate ward areas. A separate tailored email was sent to every FY1 that had completed the NHSCT blood culture training programme and this email contained a similar run chart of AAH PVC adverse event rates. In addition, information relating to any problematic PVC identified to a practitioner was also provided, customised to the specific individual only. Information supplied included date of PVC insertion, ward in which it was carried out and adverse event identified. There was no sharing of problematic PVC practitioner data with managers or among practitioner groups.

\section{Statistical analysis}

Statistical process control (SPC) was used to analyse the performance of the PVC insertion process using time series data, through the use of control p-charts [27]. Impact of the study was assessed using pre- and post- intervention analysis of the mean PVC Clinical Adverse Events (AE) rate and linear regression modelling. A Statistical Process Control p-chart was constructed using the PVC complications over the 72-week study. Due to the variation in sample size, variable upper and lower control limits were established with each observation plotted against its own control limit in a stair-step manner representing \pm 3 standard deviations [28]. All statistical analysis was performed using Minitab 17 (2010) statistical software (Minitab Inc., USA). Each study phase was described by the mean proportion defective $(\overline{\mathrm{P}})$, representing the mean adverse event rate. Fisher's exact test was used to compare the proportions between two subgroups and study characteristics described for the multiple phases using ANOVA. For continuous variables, Pearson correlation and linear regression models were constructed and used to evaluate whether the interventions resulted in a statistically significant associations. Descriptive analysis of continuous data was performed $(\mathrm{P}<0.05$ was the level of significance).

\section{Results and Discussion}

A total of 4,101 PVCs were followed to completion, accounting for 2,063 patients and 13,240 catheter days. The overall study contained 949 (46\%) male and 1,114 (54\%) female patients with a median patient age of 73 , age range $18-103$. The median length indwelling time for PVC was 3 days (interquartile range [IQR]: 1-4). The combined p-chart Figure 1 gives a visual representation of the decrease in mean PVC clinical adverse events throughout the three phases of the intervention study, with control limits. All three phases show a consistent fluctuation through their respective mean line, with no obvious outlying points (Figure 1).

Phase A data appears to have the widest spread from the mean (12.84\%), indicating a greater inconsistency with the PVC procedural outcomes. Phase B has an initial tight grouping around the mean line for the first 8 weeks (9.48\%), indicating a procedural consistency when the PVC procedural kit was first introduced. Greater variation occurs through the subsequent Phase B weeks, possibly indicating a relaxation of procedural technique. Phase $\mathrm{C}$ data spread is in a tighter spread around the mean line (5.94\%), apart from the lowest clinical adverse events recorded of zero on 5 week (Sample 52). 


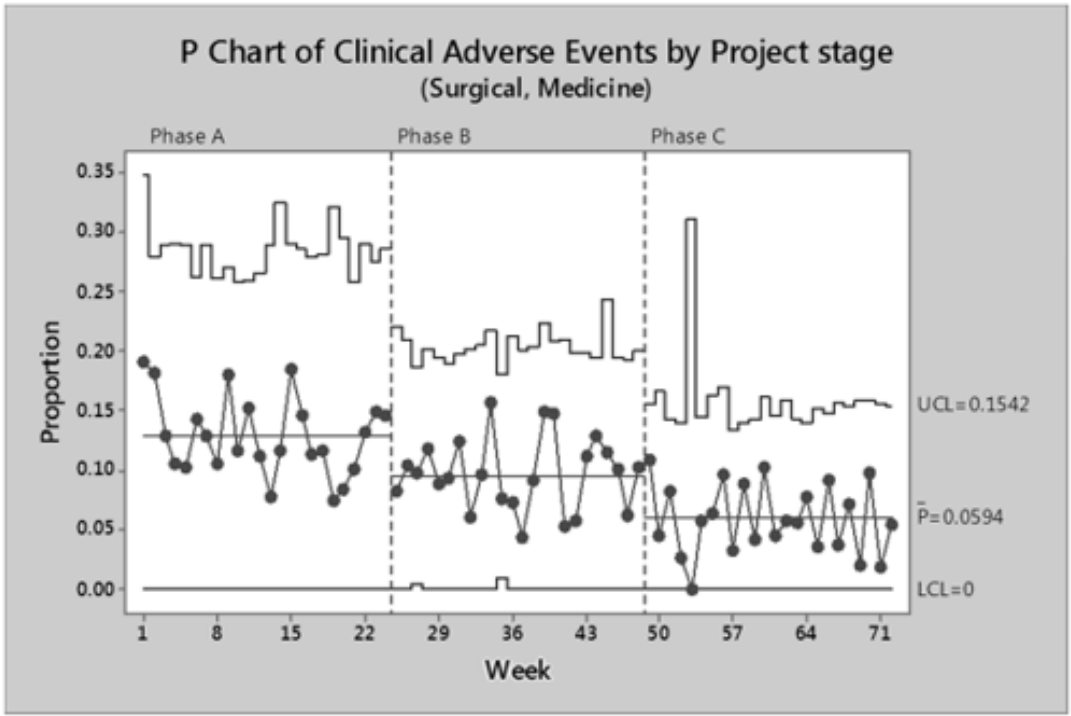

Figure 1: Summary p-chart of the weekly PVC clinical adverse event rate for the study wards (Surgical and Surgical/ Medical), over the 72-week study period, with study phase subgroups identified. Graph shows Upper Control Line (UCL), Lower Control Line (LCL), and Mean proportion $(\bar{P})$.

A Fisher's exact test for a difference of mean PVC clinical adverse event rate between Phase A 12.84\% (95\% CI: 10.86 - 15.03) and Phase B of $9.48 \%$ (95\% CI: 8.10 - 11.00) showed a statistically significant improvement $(\mathrm{p}=0.008)$. Similarly, there was another significant improvement between the PVC clinical adverse event rate of Phase B of 9.48\% (95\% CI: $8.10-11.00$ ) and Phase C of 5.94\% (95\% CI: $4.78-7.30$ ), $\mathrm{P}<0.001$. The combination of best practice guidelines, procedural kit with an enhanced training programme and feedback mechanism achieved and maintained optimal procedural practice.

Additional statistical process capability analysis was centred on phlebitis, as it is the most common clinical adverse event, seen in Figure 2. A Binomial Process Capability Report was constructed for each of the Phlebitis study phases, describing an acceptable fit to the data with all processes in statistical control. A Fisher's exact test for a difference of mean phlebitis rates between Phase A: 7.14\% (95\% CI: 5.56 - 8.88) and Phase B: 5.57\% (95\% CI: 4.50 -
6.79) showed no statistically significant improvement ( $p=0.089$ ). However, there was a statistically significant reduction in the mean phlebitis rate between Phase B: 5.57\% (95\% CI: $4.50-6.79)$ and Phase C: $3.15 \%$ (95\% CI: $2.30-4.19), p=0.047$ (Figure 2).

Throughout the 72-week study there appears to be a wide fluctuation of data points across mean lines (Figure 2). A particularly large variation can be seen in Phase A, where an unusually high rate is detected in week 15 , compared to the mean line (7.14\%). The introduction of PVC procedural kits (intervention i) seemed to have an immediate effect on phlebitis rate, with zero defects detected on week 27. Phase B then appears to stabilise about the mean line (5.57\%) for the remaining weeks. The Phase C (Intervention ii) feedback mechanism also has an immediate reduction in phlebitis rates, with four weeks recording zero events. The last 6 weeks of the study displays a very stabilised process with little variation about the mean line (3.15\%) and a final run of samples all below the $5 \%$ phlebitis target (Figure 2).

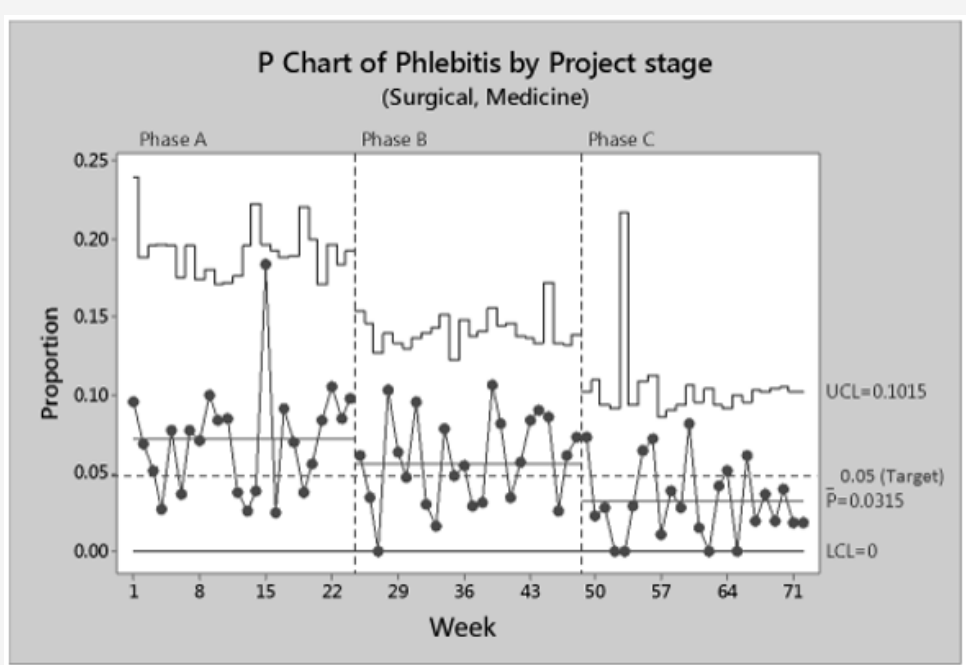

Figure 2: Summary p-chart of the weekly phlebitis rate for the study wards (Surgical and Surgical/ Medical), over the 72-week study period, with study phase subgroups identified. Graph shows Upper Control Line (UCL), Lower Control Line (LCL) and Mean proportion ( $\bar{P})$. 
Statistical process capability analysis was also used to describe the effect of study phase on non-clinical adverse events, a combination of PVC dislodgements and leaking (Figure 3). A Binomial Process Capability Report was constructed for each nonclinical adverse event study phase, describing an acceptable fit to data with all processes in statistical control. Individual reports calculated mean PVC non-clinical adverse event rates as follows; Phase A: $22.35 \%$ (95\% CI: 19.70 - 24.86), Phase B: 31.80\% (95\%
CI: 29.55 - 34.12) and Phase C: $25.66 \%$ (95\% CI: 23.42 -28.01). Phase A (baseline) data show a $22.35 \%$ mean failure rate, with a significant increase to $32.14 \%$ on the introduction of PVC procedural kits for Phase B. Phase C feedback shows a significant decrease in the mean PVC non-clinical adverse event rate of $25.66 \%$ when compared to Phase B, however it has only been reduced back to the initial pre- intervention baseline rate.

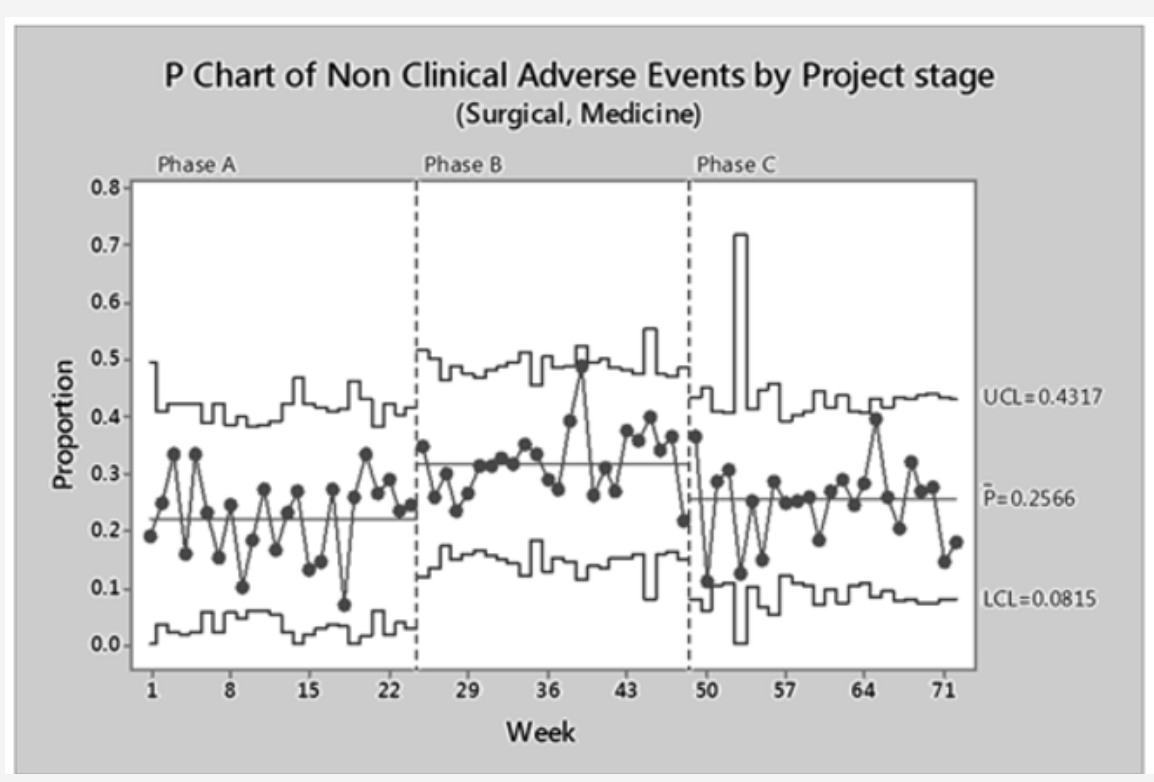

Figure 3: Summary p-chart of the weekly PVC non-clinical adverse event rate for the study wards (Surgical and Surgical/ Medical), over the 72-week study period, with study phase subgroups identified. Graph shows Upper Control Line (UCL), Lower Control Line (LCL) and Mean proportion $(\bar{P})$.

A Pareto chart was developed to identify the documented reason for PVC removal through the course of the study period, as seen in Figure 4 . From the chart only $63.7 \%$ of PVC devices are being intentionally removed when they are no longer required.
An important note is that no PVC related Blood Stream Infections (PVC BSI) were identified throughout the course of the PVC study. However, surprisingly $27.2 \%$ of PVCs are removed due to nonclinical adverse events; dislodgements and leaking (Figure 4).

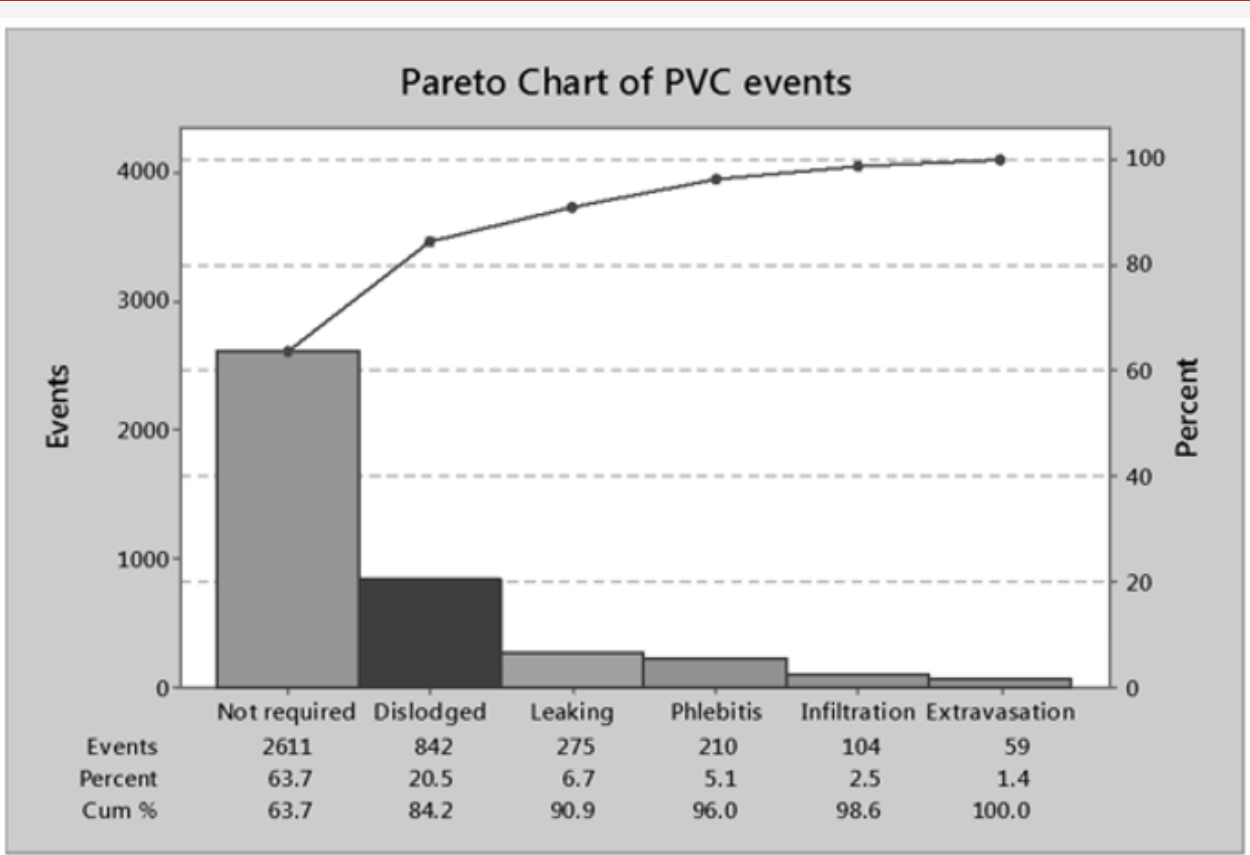

Figure 4: Pareto chart displaying all known causes of PVC removal over the complete 72 week study period. 


\section{Conclusion}

The consequence of a harmful PVC in situ can have a negative impact on patient care, practitioner time and overall hospital financial cost [7]. From the initial baseline phase of the study the overall PVC adverse event rate was very high at $35.04 \%$, resulting in over a third of devices having to be removed early due to complications. While this is similar to some reported failure rates of $41 \%$ and above in comparable patient populations, it is considered unacceptable in terms of patient risk and cost $[10,29,30]$. The incidence of PVC related Blood Stream Infection (PVC- BSI) remained zero throughout the course of the 72-week study, which would indicate a very low or negligible risk of a PVC-BSI event under current procedural training and guidelines in place [7].

In relation to other clinical adverse events, Phase A baseline assessment reported phlebitis, infiltration and extravasation all above acceptable complication rates found in clinical practice $[10,30]$. Evidence-based care bundle guidelines for PVC insertion were an established practice within AAH prior to commencement of the study, however there was no regular monitoring system for PVC related complications in use. The absence of surveillance may have led to a lack of awareness regarding high PVC complication rates and would have suggested prevalent non-compliance with guidelines in place [22].

In addition, individual collection of procedural components was at practitioner discretion. While use of evidenced-based care bundles have been shown to be effective in reducing PVC complications, compared to areas with no standard guidelines in place, there are limitations regarding long term effectiveness as a singular strategy $[1,22]$. The most concerning result from the Phase A data is the large number of PVC failures due to Non-Clinical Adverse Events (Non-Clinical AE). These are in line with the higher rates reported in the literature by Jackson (2012) at 36\% [17]. The main reason identified for PVC failure due to Non-Clinical AE is the securement dressing [31]. The dressing used for the duration of Phase A did conform to all required standards for use in an NHS clinical setting, thus implying improper technique may be the major contributing factor [31]. Introduction of PVC procedural kits and associated training had a significant impact on combined PVC clinical adverse events rate. The main advantage of procedural kits is to ensure that essential equipment is available for PVC insertion each time, thereby removing unwarranted variation, increasing compliance with care bundle guidelines and allowing practitioners to focus on technical elements of procedures [2,27]. Prior to the intervention, practitioners had to gather equipment from various stock locations, which could have contributed to lack of procedural consistency resulting in poor Phase A PVC Clinical AE rates [22].

In relation to phlebitis, although there was a reduction, the Phase B intervention did not have a significant impact on rates of phlebitis compared to baseline results. The mean phlebitis rate remained above the acceptable 5\% PVC rate during that phase. This could be explained by the increase in Non-Clinical AEs, which occurred during the same time period. Mechanical phlebitis can result from micro-movement of the PVC causing friction within the vein [31]. Curran et al. (2000) found different PVC dressings are not a significant factor in the development of phlebitis, however phlebitis can be linked to the inappropriate placement or improper securement of the device. Phase B did have a particular problem with Non-Clinical AEs, which may have had a knock-on effect for phlebitis rates, limiting the Phase B intervention.

The PVC procedural kits were introduced along with enhanced training to include greater emphasis on insertion technique through a more interactive approach [24]. While it is difficult to assess the direct impact of training alone, it must be considered as part of the procedural kits intervention. According to the literature there would have been limited benefit from introducing enhanced training as a single intervention, because of the high-quality training already in place [21]. However the focused training and lecture bundle provided a convenient, time-saving and inexpensive method to target all relevant participants prior to OSCE assessment.

The Phase B intervention did have a negative impact on NonClinical AEs, resulting in a significant increase in the associated rates. This was an unexpected negative impact of introducing a new procedural kit into clinical practice. The securement dressing in the PVC procedural kit again conformed to all the required standards for use in an NHS clinical setting and its use was covered as part of the training provided. The application of PVC securement dressings may highlight a limitation of simulation training, as the synthetic rubber arm enables easy application, which is in contrast to the real-world setting. A greater emphasis on device securement may have had an effect on the subsequent failure rates. Statistical process control enables a system for both assessing change of incontrol processes using evaluative methodologies and detecting extreme variation within a process using near real-time analysis [32]. The study used real-time run charts for the dissemination of monthly PVC complication results, which are simple to update and straightforward to interpret and one of the major advantages of using the SPC method [33]. The p-chart combines time series analysis with a basic presentation of data and is a commonly used tool to monitor adverse events for quality improvement initiatives in clinical practice [32,33]. Using p-chart tracking of monthly rates makes it possible to monitor for spikes, such as setting a $5 \%$ benchmark for phlebitis, as suggested by Curran et al. [34].

The addition of continuous feedback of results did have a significant impact on both the combined Clinical AE and individual phlebitis rates, similar to other published studies in areas that had appropriate training and care bundles in place $[33,34]$. The feedback of results enables management to highlight particular areas of interest to relevant staff, maintain awareness of the situation and motivate by setting and achieving targets [34]. The feedback mechanism augmented the previous intervention (procedural kit and enhanced training) to reduce phlebitis rates below the gold standard 5\% benchmark. Improvements were eventually seen in the Non-Clinical AEs, although rates only returned to preintervention levels. 
The study had some limitations. One of the main limitations of this research is the lack of a control group to further strengthen the study design and impact of results. Due to the standardized training and multiple rotations of medical staff within the NHSCT, this was not possible. Another limitation of the SPC study design is the unaccounted secular trend, highlighted by the Standards for Quality Improvement Reporting Excellence guidelines [33,35]. There were no observed explanations, other than intentionally introduced interventions, to account for the PVC adverse event rate outcomes recorded.

In conclusion, the study demonstrated the benefit of introducing PVC procedural kits with an enhanced training programme to significantly reduce clinical adverse events, compared to the previous standard practice. The addition of a feedback mechanism to give a general overview of current PVC adverse event rates, in addition to focused individual feedback further reduced the serious complications associated with the procedure. The Statistical Process Control method was an appropriate tool to initially highlight poor and previously unknown complications resulting from PVC procedures.

\section{Acknowledgement}

The authors would like to acknowledge Iskus Health Ltd for their funding of the research. Also, all the staff at Antrim Area Hospital who contributed to the study.

\section{Conflict of Interest}

While contributors from Iskus Health Ltd had a financial interest in the study, there was no conflict of interest in the research governance and conduct of the research, nor in the reporting arrangements.

\section{References}

1. Boyd S, Aggarwal I, Davey P, Logan M, Nathwani D (2011) Peripheral intravenous catheters: the road to quality improvement and safer patient care. J Hosp Infect 77(1): 37-41.

2. Franklin BD, Deelchand V, Cooke M, Holmes A, Vincent C (2012) The safe insertion of peripheral intravenous catheters: a mixed methods descriptive study of the availability of the equipment needed. Antimicrob Resist Infect Control 1(1): 15-20.

3. Castro-Sánchez E, Charani E, Drumright LN, Sevdalis N, Shah N, et al. (2014) Fragmentation of care threatens patient safety in peripheral vascular catheter management in acute care--a qualitative study. PLoS One 9(1): e86167.

4. Bolton D (2010) Improving peripheral cannulation practice at an NHS Trust. BJN 19(21): 1347-1350.

5. Marsh N, Webster J, Flynn J, Mihala G, Hewer, et al. (2015) Securement methods for peripheral venous catheters to prevent failure: a randomised controlled pilot trial. J Vasc Access 16(3): 237-244

6. Alexandrou E, Ray-Barruel G, Carr PJ, Frost S, Inwood S, et al. (2015) International prevalence of the use of peripheral intravenous catheters. J Hosp Med 10(8): 530-533.

7. Zingg W, Pittet D (2009) Peripheral venous catheters: an underevaluated problem. Int J Antimicrob Ag 34(4): 38-42.

8. Gallant P, Schultz AA (2006) Evaluation of a visual infusion phlebitis scale for determining appropriate discontinuation of peripheral intravenous catheters. J Infus Nurs 29(6): 338-345.
9. Helm RE, Klausner JD, Klemperer JD, Flint LM, Huang E (2015) Accepted but unacceptable: peripheral IV catheter failure. J Infus Nurs 38(3): 189203.

10. Abolfotouh MA, Salam M, Bani-Mustafa A, White D, Balkhy HH (2014) Prospective study of incidence and predictors of peripheral intravenous catheter-induced complications. Ther Clin Risk Manag 10(1): 993-1001.

11. Wallis MC, McGrail M, Webster J, Marsh N, Gowardman J, et al. (2014) Risk factors for peripheral intravenous catheter failure: a multivariate analysis of data from a randomized controlled trial. Infect Cont Hosp Ep 35(1):63-68.

12. Maki DG, Kluger DM, Crnich CJ (2006) The risk of bloodstream infection in adults with different intravascular devices: a systematic review of 200 published prospective studies. Mayo Clin Proc 81(9):1159-1171.

13. Al-Benna S, O’Boyle C, Holley J (2013) Extravasation injuries in adults. ISRN Dermatol 856541

14. White SA (2001) Peripheral intravenous therapy-related phlebitis rates in an adult population. J Infus Nurs 24(1): 19-24.

15. Gupta A, Mehta Y, Juneja R, Trehan N (2007) The effect of cannula material on the incidence of peripheral venous thrombophlebitis. Anaesthesia 62(11): 1139-1142.

16. Dychter SS, Gold DA, Carson D, Haller M (2012) Intravenous therapy: a review of complications and economic considerations of peripheral access. J Infus Nurs 35(2): 84-91.

17. Jackson A (2012) Retrospective comparative audit of two peripheral IV securement dressings. BJN 21(2):1-4.

18. O'Grady NP, Alexander M, Burns LA, Dellinger EP, Garland J, et al. (2011) Guidelines for the prevention of intravascular catheter-related infections. Clin Infect Dis 52(9): 162-193.

19. Lolom I, Deblangy C, Capelle A, Guerinot W, Bouvet E, et al. (2009) Effect of a long-term quality improvement program on the risk of infection related to peripheral venous catheters. Press Med 38(1): 34-42.

20. Freixas N, Bella F, Limón E, Pujol M, Almirante B, et al. (2013) Impact of a multimodal intervention to reduce bloodstream infections related to vascular catheters in non-ICU wards: a multicentre study. Clin Microb Infec 19(9):838-844.

21. Morse L, McDonald M (2009) Failure of a poster-based educational programme to improve compliance with peripheral venous catheter care in a tertiary hospital. A clinical audit. J Hosp Infect 72(3): 221-226.

22. Johansson ME, Pilhammar E, Khalaf A, Willman A (2008) Registered nurses' adherence to clinical guidelines regarding peripheral venous catheters: a structured observational study. Worldviews Evid Based Nurs 5(3): 148-159.

23. Parras F, Ena J, Bouza E, Guerrero MC, Moreno S, et al. (1994) Impact of an educational program for the prevention of colonization of intravascular catheters. Infect Cont Hosp Ep 15(4): 239-242.

24. Fenik Y, Celebi N, Wagner R, Nikendei C, Lund F, et al. (2013) Prepackaged central line kits reduce procedural mistakes during central line insertion: a randomized controlled prospective trial. BMC Med Educ 13(60): $1-7$

25. Bamber AI, Cunniffe JG, Nayar D, Ganguly R, Falconer E, (2009) Effectiveness of introducing blood culture collection packs to reduce contamination rates. Brit J Biomed Sci 66(1): 6-9.

26. Department of Health (2011) High Impact Intervention: Peripheral intravenous cannula care bundle. London: Crown [Online].

27. Fretheim A, Tomic 0, (2015) Statistical process control and interrupted time series: a golden opportunity for impact evaluation in quality improvement. BMJ Qual Saf 24(12): 748-752.

28. Benneyan J, Lloyd R, Plsek P (2003) Statistical process control as a tool for research and healthcare improvement. Qual Saf Health Care 12(6): 458-464.

29. Pasalioglu KB, Kaya H (2014) Catheter indwell time and phlebitis development during peripheral intravenous catheter administration. Pak J Med Sci 30(4):725-30. 
30. Webster J, Osborne S, Rickard CM, Marsh N (2019) Clinically-indicated replacement versus routine replacement of peripheral venous catheters. Cochrane Db Syst Rev 5:CD007798.

31. Marsh N, Webster J, Mihala G, Rickard CM (2015) Devices and dressings to secure peripheral venous catheters to prevent complications. Cochrane Db Syst Rev 6: CD011070.

32. Timmerman T, Verrall T, Clatney L, Klomp H, Teare G (2010) Taking a closer look: using statistical process control to identify patterns of improvement in a quality-improvement collaborative. Qual Saf Health Care 19(1):1-6.
33. Hagiwara AM, Gäre AB, Elg M (2016) Interrupted Time Series Versus Statistical Process Control in Quality Improvement Projects. J Nurs Care Qual 31(1): 1-8.

34. Curran E, Coia J, Gilmour H, McNamee S, Hood J (2000) Multi-centred research surveillance project to reduce infections/phlebitis associated with peripheral vascular catheters. J Hosp Infect 46(3): 194-202.

35. Ogrinc G, Davies L, Goodman D, Batalden P, Davidoff F, et al. (2016) SQUIRE 2.0 (Standards for Quality Improvement Reporting Excellence): revised publication guidelines from a detailed consensus process. J Nurs Care Qual; 31(1): 1-8. 